While awaiting experiments destined to throw light on the still obscure question of the mode of action of serums, we give preference to the theory propounded by Metschnikoff, who looks upon them not as antitoxic but as stimulating agents of phagocytosis; in other words, as stimulines, provokers of organic resistance.

Therefore, as we admit that the microbial destruction and the arrest of infection are due to phagocytosis, the aim of our therapy must be to increase the activity of the phagocytes, in order that they may the more easily accomplish their mission. Moreover, the happy results that we have obtained this winter by means of natural serotherapy lead us to believe that this is the real and only effective method of realizing the cure of tuberculosis, especially in its early stages, now that the means of diagnosis which we possess permit us to discover the very earliest symptoms of incipient tuberculosis.

This treatment is absolutely innocuous and easily applied; one hypodermic injection of $2 \mathrm{cc}$. every other day. In some cases, however, in the case of nerve patients especially, I have observed after each injection an exaggeration of cellular activity, showing itself in the shape of fever, erythema and dyspnea; in such cases I administer the serum internally. But, in order to obtain the same tonic and stimulating effects, $I$ have to increase the dose and administer $10 \mathrm{cc}$. instead of 2 , as in the case of hypodermic injections.

These results agree with those obtained by Grasset, who concludes by saying that the administration of serum internally is the method of choice, because it is free from danger and gives rise to no accidents. Nevertheless, even with Bertin's serum, we are of opinion that the cacodylate medication should be employed as a precious adjuvant in most cases on the same level as tannin, iodine and cod liver oil.

\section{THE CHEMICAL PROPERTIES OF LEUCO- CYTES.}

BY EDWARD T. WILLIAMS, M.D., Boston.

The subdivision of the leucocytes by Ehrlich and his followers into over half a dozen different species, distinguished mainly by their reaction to staining agents, makes their study a somewhat difficult task. This difficulty is increased to the common mind by the artificial nomenclature adopted by recent writers on the subject. Professor Ewing of New York, in his late work on the blood, ${ }^{1}$ divides the leucocytes into three classes, basophile, neutrophile and oxyphile. The basophiles are distinguished by their affinity for alkaline dyes, the neutrophiles are indifferent, while the oxyphiles stain only with acid dyes, like eosin, or acid-fuchsin (Ehrlich's triple stain).

Now basophile, base-loving, means in plain English acid; for that which loves a base is acid. Oxyphile, acid-loving, means alkaline. Neutro1 Clinical Pathology of the Blood; Lea Brothers \& Co., Phila-
delphia, 1901. phile is neutral. It would seem wise, therefore, to change the names of Professor Ewing's three classes and say simply, acid, neutral and alkaline leucocytes. This assists comprehension and fixes their staining affinities more clearly in the mind.

The nuclei of all three classes stain best with alkaline dyes, as methylene-blue, methyl-green or dahlia. They are, therefore, acid. The bodies of the normal, single-nucleated leucocytes take the same dyes. This proves them to be acid. The bodies of the normal, many-nucleated leucocytes are neutral to dyes. They come under the neutral class, which includes also the neutral myelocytes (a doubtful name), which are found only in disease. The bodies of the so-called eosinophile leucocytes and eosinophile myelocytes take only the acid dyes. They are, therefore, alkaline.

The alkaline leucocytes occur chiefly in disease. There are, to be sure, a few eosins in normal blood (about $3 \%$ of all leucocytes, according to Ewing), but any marked increase in their number is sure to be a symptom of disease. This is especially characteristic in leucocythæmia, trichiniasis and some other diseases. The iodine-staining leucocytes may be conveniently classed as alkaline, since iodine acts as an acid with alkalies. On the other hand the fodder-cells (mast-cells) may be classed with the acid group, since they stain only with Ehrlich's dahlia solution, a basic dye.

It is doubtless impossible in our present state of knowledge to explain precisely the causes of these diverse reactions. It may be possible to make a plausible guess. We have seen that all nuclei àre acid. They owe this property, without doubt, to the nuclein which they contain. Nuclein is acid. When boiled with alkalies it yields phosphoric acid. ${ }^{2}$ Phosphoric acid, it may be remarked, is the only mineral acid which does not coagulate albumin. It is the presence of this acid undoubtedly which makes nuclein acid. According to the experiments of Kossel, quoted by Vaughan and Novy, ${ }^{3}$ nuclein when boiled with acids yields certain organic, albuminoid bases, as adenine, sarcine, xanthine, spermine and others. They belong to the numerous class of putrefactive alkaloids invariably produced by the decomposition of albuminous or proteid substances whether animal or vegetable. We must conceive, therefore, of nuclein as some sort of a phospho-albumin, whose composition has not been precisely determined. Probably there are many nucleins. It may be reasonably conceived that the cell bodies of the acid, single-nucleated leucocytes also contain nuclein. These form over $20 \%$ of the whole number of leucocytes (Ewing). They are probably the youngest of the leucocytes, since they are formed mainly in the lymph glands, and according to the old doctrine ultimately develop into multinuclears by division of the nucleus. Ewing rejects this hypothesis; yet it is hard to conceive, as he seems to do, that every variety of leucocyte is a fixed and definite anatomical ele-

2 Merck's Index, New York, 1896; sub voce.

3 Ptomaines and Leucomaines; Lea Brothers \& Co., Philadelphia, 1891 . 
ment: If the uninuclears contain nuclein, their affinity for basic dyes is readily understorid.

The cell bodies of the multinuclears are neutral to dyes. Hence nuclein is absent. If ever present it has disappeared, possibly as the result of decomposition. Warren, ${ }^{4}$ Cornil and Ranvier, ${ }^{5}$ Wharton Jones ${ }^{6}$ and other authorities have declared their opinion that the multinuclears are in a state of decomposition.

The alkaline leucocytes certainly seem to be $\mathrm{dy}$ ing forms. They are almost invariably the products of disease. We know that leucocytes (like other cells) are subject to degeneration. 'The fatty degeneration is extremely common. This is constantly seen in suppurative processes. The cells are full of oil drops and finally dissolve. Ewing admits seeing this himself and finding the oil drops blackened, as might be expected under osmic acid. Amyloid degeneration may very likely be one of the causes of the peculiar iodine staining of certain morbid leucocytes called by 'T. Dunham of New York ${ }^{7}$ iodophiles. Ewing calls this the glycogen reaction; still he considers it due to degeneration of the cell substance. Iastly, the eosinophiles, as previously remarked, are mainly the products of disease. They are often associated with spermine (Charcot-Leyden) crystals, which sometimes appear either in or upon them. ${ }^{8}$ This association has been particularly noticed in leucocythæmia and asthma. Xanthine, too, is a common finding in leucocythæmia. Now spermine and xanthine, as already mentioned, are products of the decomposition of nuclein. Hence it seems rational to infer that the eosinophiles also are undergoing decomposition. The cellular protoplasm is breaking up and resolving itself into nitrogenous alkaloids. Hence their alkalinity; hence their peculiar affinity for acid dyes. For these reasons I make bold to include all alkaline (oxyphile) leucocytes under the designation of necrotic cells.

At a future period I hope to bring forward some further evidence in support of this conclusion.

\section{Medital Orogrega.}

\section{REPORT ON DERMATOLOGY.}

BY JOHN T. BOWEN, M.D., BOSTON.

ARSENICAL MELANODERMA.

Gaucher and Bernard ${ }^{1}$ reported at the Sociéte de Dermatologie et de Syphiligraphie the case of a woman of 32 who consulted them on account of a brown pigmentation, which was scattered over a large part of the body. She had first no-

4 Surgical Pathology; Saunders, Philadelphia, 1895, p. 160.

5 Pathological Histology; Lea Brothers \& Co., Philadelphia, 1880, p. 21.

T The Blood Corpuscle in Its Different Phases of Development; Philosophical Transactions, 1846. 7 Iodophilia; Boston Medical and Surgical Journal, June 13,
1901 .

8 Ewing: Clinical Pathology of the Blood; Lea Brothers \& Co., Philadelphia, 1901, p. 218.

1 Ann. de Derm. et de Syph., April, 1901. ticed, 4 years previously, that her neck was becoming dark. She had had since her infancy some tendency to freckling of the face, which increased each year, but had never become excessive. Little by little the pigmentation of the neck increased, while at the same time dark spots appeared on other parts of the body subject to friction or abundant perspiration - the waist, axillæ, etc. When seen the pigmentation had extended to almost the whole surface of the body. It was made up of spots of a more or less dark brown, rounded, and of various sizes, some no larger than a pin's head, others as large as a bean. In certain places these macules are isolated and separated by intervals of sound skin; in others they are confluent and form large brown or brownish-black plagues. They are marked on the face, forming a confluent mass over the forehead. Over the back and sides of the neck the pigmentation is very marked. The trunk is deeply pigmented with a maximum of intensity about the waist, where there is a large band of pigmentation with numerous outlying macules. It is marked on the upper part of the back and in the lumbar region. The extremities are almost entirely free from pigmentation, and show only a few light-colored patches, with the exception of the thighs, where the spots are more confluent. There is no pigmentation nor abnormality of the epidermis of the palms and soles. The mucous membrane of the mouth is unaffected.

Upon inquiry it was found that, for 6 consecutive years and up to 1 year ago, she had taken continuously, with the exception only of 8 days a month, Fowler's solution in the dose of 20 drops a day, because she had been told that it would improve her health and also give her a good color.

Lotions of sublimate, etc., were employed morning and evening for several months, during which time the patient was seen at intervals, but no change could be seen in the intensity or the extent of the pigmentation. The general condition remained good.

In this case, as in those of Mathieu and Richardiere, as well as those reported by Brouardel, the pigmentation appeared first on the neck, and extended from there to the trunk and abdomen. As in other cases, also, the pigmentation was most marked on parts exposed to pressure or friction; it had a predilection for the parts covered by the clothing, and did not affect the hands and feet, and the extremities in general very little. As in other cases, again, the skin presented a peculiar spotted appearance, that has been compared to a scattering of bits of tobacco. As is usual, there was no pigmentation of the mucous membranes. The melanoderma was extremely rebellious to treatment and to the stopping of the cause. The long persistence of the pigmentation is a rule in these cases, so that sometimes there is considerable difficulty in distinguishing it from Addison's disease. The predisposition to pigmentation shown by the previous freckling is to be noted in this case. 